\title{
Association of Serum Periostin Level with Classical Bone Turnover Markers and Bone Mineral Density in Shanghai Chinese Postmenopausal Women with Osteoporosis
}

\author{
$\operatorname{Ran} \mathrm{Li}^{1,2, *}$ \\ Xiaoyi Zhu ${ }^{1,2, *}$ \\ Mengxi Zhang ${ }^{1,2, *}$ \\ Guannan Zong ${ }^{1,2}$ \\ Keqin Zhang ${ }^{1,2}$
}

'Department of Endocrinology and Metabolism, Tongji Hospital, School of Medicine, Tongji University, Shanghai, 200065, People's Republic of China; ${ }^{2}$ Institute of Osteoporosis and Metabolic Bone Diseases, School of Medicine, Tongji University, Shanghai, 200065, People's

Republic of China

*These authors contributed equally to this work
Correspondence: Keqin Zhang

Department of Endocrinology and Metabolism, Tongji Hospital, School of

Medicine, Tongji University, No. 389,

Xincun Road, Shanghai, 200065, People's

Republic of China

Tel +862I-66II 2032

Email keqzhang2018@I63.com
Background: It has been reported that serum periostin levels are significantly higher in postmenopausal patients with osteoporotic fractures. Nonetheless, the levels of serum periostin in postmenopausal women with different bone mass remain unclear.

Purpose: The objective of the study was to identify the levels of serum periostin in Chinese postmenopausal women with different bone mass, and the correlations between the periostin levels and the classical bone turnover markers (BTMs), and bone mineral densities (BMDs) at different sites.

Patients and Methods: This study enrolled 331 Chinese postmenopausal women in Shanghai; their clinical features were collected; their levels of serum periostin and traditional BTMs were measured by ELISA or the fully automated immunoassay analyzer; their BMDs at different sites were measured by dual-energy X-ray absorptiometry (DXA).

Results: According to the $T$-value of bone mineral density (BMD), these postmenopausal women were divided into normal group $(n=84)$, osteopenia group $(n=126)$ and osteoporosis group $(n=121)$. There was no significant difference in the serum periostin levels among the above three groups of subjects. In addition, Spearman correlation analysis also revealed that no correlation was observed between the value of serum periostin and those of traditional BTMs, and BMDs at different sites, respectively. The values of traditional BTMs were negatively correlated with those of BMDs at all measured sites. Furthermore, the receiver-operating characteristic (ROC) curves analysis indicated that among the periostin and traditional BTMs mentioned above, the best predictors for postmenopausal osteoporosis in Shanghai Chinese postmenopausal women were osteocalcin (OC) and procollagen type $1 \mathrm{~N}$-terminal propeptide (P1NP) [the areas under the ROC curve (AUC) $=0.746$ and 0.761 , respectively].

Conclusion: Serum periostin may not be used as a marker of systemic bone metabolism in Shanghai Chinese postmenopausal women without prior fracture. In addition, serum P1NP and OC levels may be the predictors of osteoporosis occurrence in Chinese postmenopausal women. Keywords: postmenopausal women, periostin, bone turnover markers, bone mineral density

\section{Introduction}

Osteoporosis is a systemic metabolic disease mainly characterized by bone mass loss and microarchitectural deterioration of bone tissue, resulting in increased bone fragility and prone to bone fractures. ${ }^{1}$ The pathogenesis of osteoporosis is the imbalance of bone remodeling. ${ }^{2}$ Estrogen deficiency play an important role in the occurrence of osteoporosis by increasing bone structure remodeling. ${ }^{3}$ Early 
epidemiological studies indicated that in many countries, the prevalence of osteoporosis in postmenopausal women is significantly higher than that of men. ${ }^{4-6}$ Osteoporosis and osteoporotic fractures decrease the quality of life, increase the risk of death and result in severe economic burden on families and the global economy. ${ }^{4}$ Overall, postmenopausal osteoporosis is becoming an urgent global health concern. Therefore, it is necessary to early detect and diagnose osteoporosis and to identify patients at high risk for fracture.

The World Health Organization (WHO) defines that bone mineral density (BMD), usually measured by dual-energy X-ray absorptiometry (DXA), is the diagnostic criteria for osteoporosis and the predictor of fracture risk. Osteoporosis is defined as a BMD value which is equal to or more than $2.5 \mathrm{SD}$ ( $T$-score) below the mean value of peak BMD of young woman. ${ }^{7}$ However, several studies have shown that there were several limitations of using BMD to predict fracture risk. Siris et $\mathrm{al}^{8}$ found that $82 \%$ of postmenopausal women with fractures had $T$-scores which were above -2.5 . Similarly, Schuit et $\mathrm{al}^{9}$ demonstrated that only $44 \%$ of women with nonvertebral fractures had $T$-scores which were at or below -2.5 . In general, using only BMD to diagnose postmenopausal osteoporosis (PMOP) may not accurately predict fracture risk. Therefore, if using BMD as the indicator for treatment, there may be a large proportion of women with PMOP who cannot receive timely treatment.

Due to the defect of BMD in clinical practice, researchers have explored the role of bone turnover markers (BTMs) in blood and urine, which are divided into bone formation markers and bone resorption markers. Traditional bone formation markers, such as procollagen type $1 \mathrm{~N}$-terminal propeptide (P1NP), bone-specific alkaline phosphatase (BALP) and osteocalcin (OC), are related to the activity of osteoblasts. Conversely, traditional bone resorption markers, such as cross-linked C-telopeptide of type I collagen (CTX), cross-linked N telopeptide of type I collagen (NTX) and tartrate-resistant acid phosphatase $5 \mathrm{~b}$ (TRACP $5 \mathrm{~b}$ ), reflect activity of osteoclasts. ${ }^{10}$ Vasikaran et $\mathrm{al}^{11}$ performed prospective cohort studies and concluded that high levels of BTMs may predict fracture risk in postmenopausal women independently of BMD and reflect treatment-induced dynamic changes in bone metabolism. In addition, studies have shown that the combination of BTMs and BMD will improve the risk prediction of PMOP fractures. ${ }^{12}$ However, the traditional BTMs currently in widespread use also have limitations, ie, they mainly reflect endosteal bone remodeling, but not the periosteal metabolism. ${ }^{13}$ A study has shown that periosteal metabolism also plays an important role for the maintenance of skeletal integrity. ${ }^{14}$

Periostin is an extracellular matrix protein mainly secreted by osteocytes and osteoblasts in the periosteum. ${ }^{15,16}$ Periostin has shown to play a vital role in the maintenance of bone microarchitecture and bone strength by regulating collagen crosslinking and osteoblast cell adhesion. ${ }^{17}$ Furthermore, absence of periostin gene in mice exhibited lower bone mass, lower cortical bone volume, disordered arrangement of collagen fibril and decreased bone strength, which may be related to decreased proliferation and increased apoptosis of osteoblasts. ${ }^{15,18}$ Conversely, other researchers have reported that overexpression of periostin gene in rats and osteoblastic cells increased bone formation and bone mass due to the increased osteoblast proliferation and differentiation. ${ }^{15}$ Interestingly, in a 7-year prospective analysis of the study, higher serum periostin levels have been reported to be associated with increased fracture risk in postmenopausal women from the Os des Femmes de Lyon cohort study independently of age, bone turnover markers, and BMD. ${ }^{19}$ Other clinical studies have also demonstrated that circulating periostin levels were positively correlated with non-vertebral fractures after adjustment for BMD and potential confounders in Korean postmenopausal women. ${ }^{20}$ Similarly, high periostin levels were negatively related with femoral neck BMD in Chinese older women with acute hip fractures. ${ }^{21}$ However, Anastasilakis and his colleagues ${ }^{18}$ found that there was no difference in circulating periostin levels between Caucasian postmenopausal women with normal bone mass (BMD $T$-score of $>-1.0$ ) and low bone mass (BMD $T$-score of $\leq-2.0$ ).

Traditional bone formation markers, such as P1NP, OC and BALP, are secreted by osteoblasts. ${ }^{10}$ Periostin is also highly expressed by osteoblasts and osteocytes. ${ }^{15,16}$ Therefore, we hypothesized that serum periostin may also reflect the bone formation activity, such as P1NP, OC and BALP. To date, few studies have investigated the associations of serum periostin level with those of traditional BTMs and with bone mineral densities (BMDs) at different sites respectively in Chinese postmenopausal women. Therefore, our aim was to answer the above questions by epidemiological study in Shanghai local population.

\section{Methods}

\section{Patients and Methods}

Postmenopausal women were recruited in Community Health Service Center, Ganquan Street, Putuo District, Shanghai from January 2017 to September 2018. 
According to the recommendation of the WHO, we classified BMD $T$-score [at the lumbar spine (L1-L4) and/or the left hip] $\geq-1$ as the normal group, $-2.5<T$-score $<-1$ as the osteopenia group, and $T$-score $\leq-2.5$ as the osteoporosis group. We selected the lowest BMD value among all measured sites as the value for diagnosis in every subject. The inclusion criteria of this study were as follows: 1) menopause for more than 1 year; 2) the person lived in China for more than 10 years; 3) the person signed informed consent. The exclusion criteria of this study were as follows: 1) patients with secondary osteoporosis; 2) patients with a history of fractures; 3) patients with diseases that could affect bone metabolism; 4) patients who discontinued bisphosphonates for less than 1 year and other medications that could affect bone metabolism for less than 6 months. All subjects provided informed consent to participate in this study. The study protocol and procedures conform to the ethical guidelines of the 1975 Declaration of Helsinki. The present study was approved by the Ethics Committee of Tongji Hospital, Tongji University.

\section{Laboratory Measurements}

All participants' blood samples were collected after an overnight fast and stored at $-80^{\circ} \mathrm{C}$ until analysis. All blood samples were tested by the clinical laboratory of Tongji Hospital of Tongji University. The levels of serum periostin were measured using an enzyme-linked immunosorbent assay (ELISA) kit (Wu HaoBio-Tech Co., LTD., Shanghai, China) according to the manufacturer's instructions. The intra-assay coefficient of variation $(\mathrm{CV})$ was $<10 \%$ and inter-assay $\mathrm{CV}$ was $<12 \%$. Serum BALP and TRACP $5 \mathrm{~b}$ were measured using ELISA kit (Gukang Bio-Tech Co., LTD., Guangdong, China). The intraand inter-assay CV of BALP and TRACP $5 b$ were both $\leq 10 \%$. The levels of serum P1NP, $\beta$-CTX and OC were measured using the fully automated immunoassay analyzer (Cobase 601, Roche Diagnostics Co., LTD., Germany). The intra- and interassay $\mathrm{CV}$ were $<4.5 \%$ and $<5.5 \%$ for $\mathrm{P} 1 \mathrm{NP}, \leq 4.0 \%$ and $\leq 6.0 \%$ for $\beta$-CTX, and $<5.0 \%$ and $<7.0 \%$ for OC, respectively.

\section{Bone Mineral Density Measurements}

The BMD was measured by DXA (Hologic, Bedford, MA, USA) at the lumbar spine (L1-L4) and the left hip including femoral neck, trochanter and the total hip. The results were expressed in $\mathrm{g} / \mathrm{cm}^{2}$ and $T$-score. We calibrated the instrument daily, and the same technician completed the measurement. The daily $\mathrm{CV}$ of BMD measurements in the lumbar spine model were $<1 \%$.

\section{Statistical Analysis}

All data were analyzed by the statistical package for social sciences (SPSS) version 22.0. Kolmogorov-Smirnov test assessed whether continuous variables followed a normal distribution. The data were presented as mean \pm standard deviation (SD) if it followed normal distribution. Otherwise, it was presented as the median (interquartile range). The three groups were analysed with one-way ANOVA followed by the least significant differences (LSD) or Dunnett's T3 post hoc multiple comparison test. Spearman correlation analysis was performed to explore the correlations between serum periostin and other BTMs, and between traditional BTMs and BMDs at different sites. Receiver operating characteristic (ROC) curve analysis was constructed to determine predictors for PMOP $P$-value $<0.05$ was considered statistically significant.

\section{Results}

\section{The Baseline Characteristics of the Postmenopausal Women}

The baseline characteristics of the 311 postmenopausal participants are presented in Table 1, including demographics, clinical characteristics and BMD. The participants were divided into three groups based on the BMD $T$-score: the normal group ( $\mathrm{n}=84)$, the osteopenia group $(\mathrm{n}=126)$, and the osteoporosis group $(\mathrm{n}=121)$. There were no differences among the three groups in age and menopausal age. Body weight and body mass index (BMI) were significantly lower in the osteopenia group than in the normal group. In addition, the osteoporosis group had a statistically significant lower levels of height, weight and BMI than the normal group or the osteopenia group. As expected, the BMD values at all measured sites in the osteopenia group or the osteoporosis group were significantly decreased compared with the normal group. Consistently, the BMDs of lumbar spine (L1-L4), femoral neck and total hip were lower in the osteoporosis group than those in the osteopenia group.

\section{Differences in Serum Levels of Periostin and Traditional BTMs Among the Three Groups}

For serum periostin, no significant difference was observed between the normal group, the osteopenia group and osteoporosis group (Figure 1A). 
Table I The Clinical Characteristics of the Postmenopausal Women.

\begin{tabular}{|c|c|c|c|c|}
\hline \multicolumn{2}{|l|}{ Variables } & Normal Group $(n=84)$ & Osteopenia Group $(n=\mid 26)$ & Osteoporosis Group $(n=|2|)$ \\
\hline \multicolumn{2}{|l|}{ Age (years) } & $60.5 \pm 5.2$ & $60.5 \pm 4.5$ & $61.3 \pm 4.2$ \\
\hline \multicolumn{2}{|c|}{ Menopausal age (years) } & $51.0 \pm 3.5$ & $50.3 \pm 3.5$ & $50.0 \pm 3.8$ \\
\hline \multicolumn{2}{|c|}{ Height $(\mathrm{cm})$} & $159.5 \pm 5.0$ & $159.4 \pm 5.5$ & $157.5 \pm 5.1^{* * \# \#}$ \\
\hline \multicolumn{2}{|c|}{ Body weight (kg) } & $63.7 \pm 8.5$ & $61.3 \pm 8.4^{*}$ & $56.6 \pm 7.7^{* * \# \#}$ \\
\hline \multicolumn{2}{|c|}{ BMI $\left(\mathrm{kg} / \mathrm{m}^{2}\right)$} & $25.0 \pm 2.9$ & $24.2 \pm 3.1^{*}$ & $22.8 \pm 2.9 * * \#$ \\
\hline \multirow[t]{4}{*}{ BMD $\left(\mathrm{g} / \mathrm{cm}^{2}\right)$} & LS (LI-L4) & $1.0722 \pm 0.105$ & $0.902 \pm 0.054^{* *}$ & $0.749 \pm 0.079 * * \ldots$ \\
\hline & Femoral neck & $0.821 \pm 0.106$ & $0.705 \pm 0.070 * *$ & $0.625 \pm 0.078^{* * \# \#}$ \\
\hline & Trochanter & $0.770 \pm 0.086$ & $0.64 I \pm 0.188^{* *}$ & $0.606 \pm 0.10 I^{* *}$ \\
\hline & Total hip & $0.973 \pm 0.091$ & $0.838 \pm 0.132 * *$ & $0.774 \pm 0.097^{* *} \#$ \\
\hline
\end{tabular}

Note: $* P<0.05, * * P<0.01$ vs normal group; ${ }^{\# *} P<0.01$ vs oteopenia group.

Abbreviations: LS, lumbar spine; BMI, body mass index; BMD, bone mineral density.

For bone formation markers, serum OC and P1NP levels in the osteopenia group was significantly higher than those in the normal group. Furthermore, serum BALP, OC and P1NP levels in osteoporosis group were remarkably increased compared with those of both normal group and osteopenia group (Figure 1B-D). For bone resorption markers, serum $\beta$-CTX level in the osteoporosis group was higher than those in the normal group and the osteopenia group. However, serum TRACP $5 b$ levels were not significantly different among the three groups (Figure 1E and F). Our data indicated that low BMD is associated with high BTMs in postmenopausal women.
A

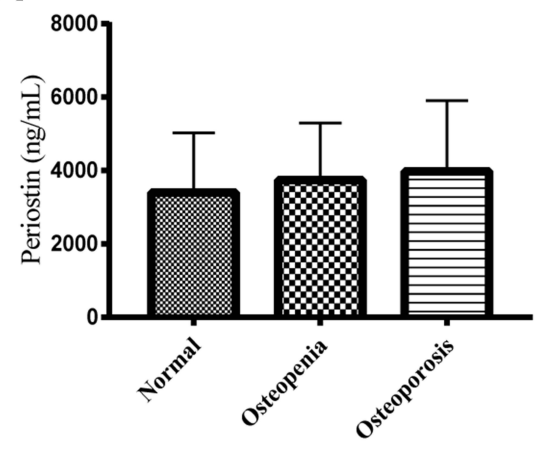

D

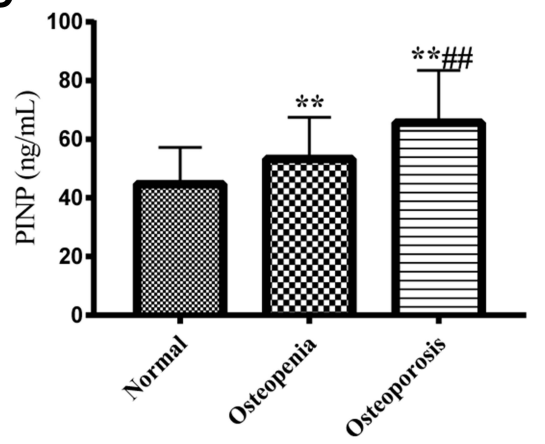

B

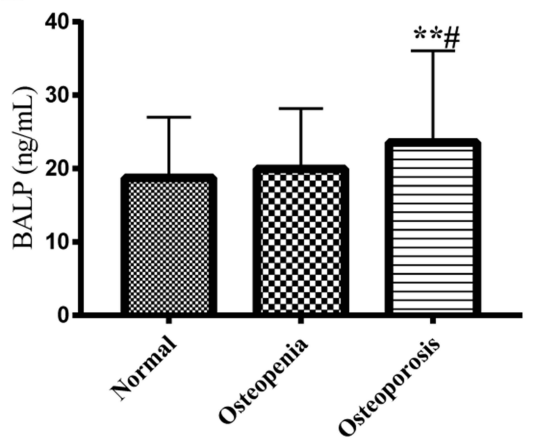

E

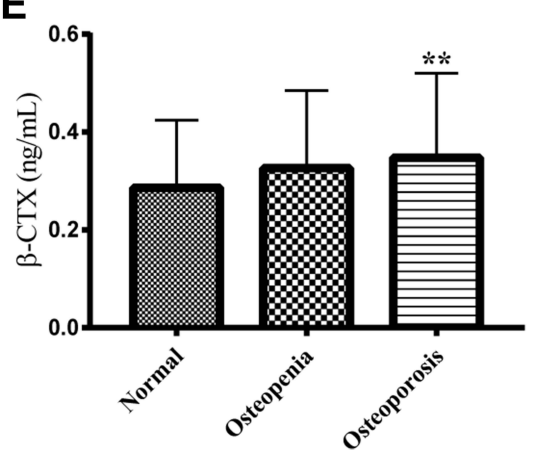

C

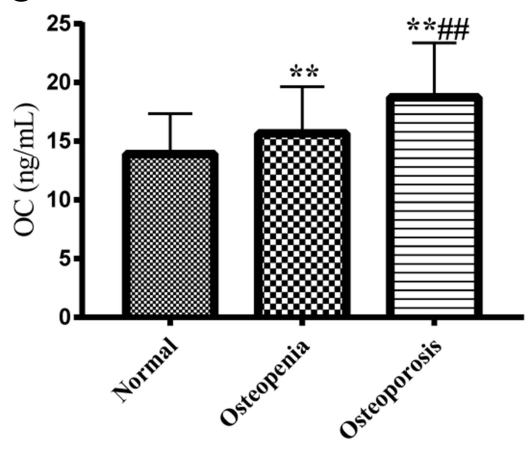

F

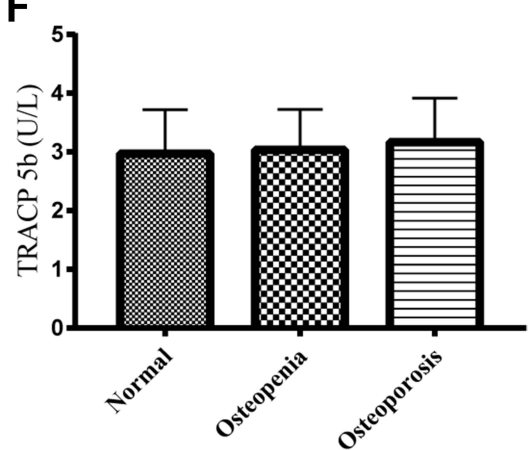

Figure I Differences in serum periostin and traditional BTMs levels among the normal group, the osteopenia group and the osteoporosis group. (A) Serum periostin levels among three groups. (B-D) Serum bone formation markers, such as BALP (B), OC (C) and PINP (D) among three groups. (E and $\mathbf{F})$ Serum bone resorption markers, such as $\beta$-CTX (E) and TRACP $5 b(\mathbf{F})$ among the three groups.

Notes: ${ }^{* * P} P<0.01$ vs normal group; ${ }^{\#} P<0.05,{ }^{\# \#} P<0.01$ vs osteopenia group.

Abbreviations: BTMs, bone turnover markers; BALP, bone-specific alkaline phosphatase; OC, osteocalcin; PINP, procollagen type I N-terminal propeptide; $\beta$-CTX, $\beta$ cross-linked C-telopeptide of type Icollagen; TRACP 5b, tartrate-resistant acid phosphatase 5b. 
Table 2 Spearman Correlations Between Serum Periostin Levels and Traditional BTMs or BMDs at Various Sites Among Postmenopausal Women Studied.

\begin{tabular}{|c|c|c|c|c|c|c|c|c|c|}
\hline & $\begin{array}{l}\text { BALP } \\
\text { (ug/mL) }\end{array}$ & $\begin{array}{l}\text { OC } \\
\text { (ng/mL) }\end{array}$ & $\begin{array}{l}\text { PINP } \\
\text { (ng/mL) }\end{array}$ & $\begin{array}{l}\beta \text {-CTX } \\
\text { (ng/mL) }\end{array}$ & $\begin{array}{l}\text { TRACP } \\
5 b(U / L)\end{array}$ & $\begin{array}{l}\text { Lumbar Spine } \\
(\mathrm{LI}-\mathrm{L} 4)\left(\mathrm{g} / \mathrm{cm}^{2}\right)\end{array}$ & $\begin{array}{l}\text { Femoral Neck } \\
\left(\mathrm{g} / \mathrm{cm}^{2}\right)\end{array}$ & $\begin{array}{l}\text { Trochanter } \\
\left(\mathrm{g} / \mathrm{cm}^{2}\right)\end{array}$ & $\begin{array}{l}\text { Total Hip } \\
\left(\mathrm{g} / \mathrm{cm}^{2}\right)\end{array}$ \\
\hline$\gamma$ & 0.093 & -0.021 & 0.030 & -0.050 & -0.040 & $-0.07 I$ & 0.002 & 0.041 & 0.043 \\
\hline$P$ & 0.102 & 0.721 & 0.612 & 0.384 & 0.481 & 0.217 & 0.969 & 0.515 & 0.456 \\
\hline
\end{tabular}

Note: Statistical significance at the $P<0.05$ level.

Abbreviations: BTMs, bone turnover markers; BMDs, bone mineral densities; BALP, bone-specific alkaline phosphatase; OC, osteocalcin; PINP, procollagen type I $\mathrm{N}$-terminal propeptide; $\beta$-CTX, $\beta$ cross-linked C-telopeptide of type I collagen; TRACP $5 \mathrm{~b}$, tartrate-resistant acid phosphatase $5 \mathrm{~b}$.

\section{The Associations Between Serum Periostin Levels and Classic BTMs or BMDs at All Measured Sites}

As shown in Table 2, there was no significant association of serum periostin with traditional BTMs, including BALP, OC, P1NP, $\beta$-CTX and TRACP 5b. In addition, circulating periostin concentration was not significantly associated with BMDs at all different sites, including lumbar spine (L1-L4), femoral neck, intertrochanter and total hip.

\section{The Association Between Classic BTMs and BMDs at All Different Sites}

The correlation between traditional BTMs and BMDs at all different sites in the postmenopausal women was shown in Table 3. Bone formation markers such as BALP, OC and P1NP and bone resorption markers such as $\beta$-CTX and TRACP $5 b$ were inversely correlated with the BMD values in all different sites.

\section{ROC Curves}

ROC curve was established, and the area under the ROC curves (AUCs) of serum periostin and traditional BTMs was observed (Figure 2). Serum OC and P1NP could better distinguished the $\mathrm{PMOP}$ patients from the nonPMOP subjects $[\mathrm{AUC}=0.746$ for $\mathrm{OC}, P<0.001,95 \%$ confidence interval (CI): $0.686-0.805$; $\mathrm{AUC}=0.761$ for $\mathrm{P} 1 \mathrm{NP}$, $P<0.001$, 95\% CI: 0.702-0.821].

\section{Discussion}

Periostin is a $93.3 \mathrm{kD}$ secreted extracellular matrix protein (in humans), named for its high levels of expression in the periosteum of long bones. ${ }^{15}$ In bone, periostin is mainly expressed by periosteal osteoblasts and osteocytes. ${ }^{16}$ Its expression level is controlled by growth factors such as transforming growth factor $\beta$ (TGF- $\beta$ ) and bone morphogenetic protein 2 (BMP2), cytokines such as tumor necrosis factor $\alpha$ (TNF- $\alpha$ ), interleukin (IL)-4 and IL-13, mechanical stimuli and parathyroid hormone (PTH). All of these factors play important roles in the regulation of

Table 3 Spearman Correlations Between Traditional BTMs and BMDs at Various Sites Among All Participants Studied.

\begin{tabular}{|c|c|c|c|c|c|}
\hline & & $\begin{array}{c}\text { Lumbar Spine } \\
\text { (LI-L4) } \\
\left(\mathrm{g} / \mathrm{cm}^{2}\right)\end{array}$ & $\begin{array}{c}\text { Femoral Neck } \\
\left(\mathrm{g} / \mathrm{cm}^{2}\right)\end{array}$ & $\begin{array}{c}\text { Trochanter } \\
\left(\mathrm{g} / \mathrm{cm}^{2}\right)\end{array}$ & $\begin{array}{c}\text { Total Hip } \\
\left(\mathrm{g} / \mathrm{cm}^{2}\right)\end{array}$ \\
\hline BALP $(\mu g / m L)$ & $\begin{array}{l}\gamma \\
P\end{array}$ & $\begin{array}{c}-0.141 * \\
0.013\end{array}$ & $\begin{array}{l}-0.276^{*} \\
<0.001\end{array}$ & $\begin{array}{l}-0.26 I^{*} \\
<0.001\end{array}$ & $\begin{array}{c}-0.203^{*} \\
<0.001\end{array}$ \\
\hline$O C(n g / m L)$ & $\begin{array}{l}\gamma \\
P\end{array}$ & $\begin{array}{l}-0.405^{*} \\
<0.001\end{array}$ & $\begin{array}{l}-0.464^{*} \\
<0.001\end{array}$ & $\begin{array}{l}-0.493^{*} \\
<0.001\end{array}$ & $\begin{array}{l}-0.44 I^{*} \\
<0.001\end{array}$ \\
\hline PINP (ng/mL) & $\begin{array}{l}\gamma \\
P\end{array}$ & $\begin{array}{l}-0.442^{*} \\
<0.001\end{array}$ & $\begin{array}{l}-0.457^{*} \\
<0.001\end{array}$ & $\begin{array}{l}-0.494 * \\
<0.001\end{array}$ & $\begin{array}{l}-0.458^{*} \\
<0.001\end{array}$ \\
\hline$\beta-\mathrm{CTX}(\mathrm{ng} / \mathrm{mL})$ & $\begin{array}{l}\gamma \\
P\end{array}$ & $\begin{array}{c}-0.153^{*} \\
0.007\end{array}$ & $\begin{array}{l}-0.316^{*} \\
<0.001\end{array}$ & $\begin{array}{l}-0.375^{*} \\
<0.001\end{array}$ & $\begin{array}{l}-0.286^{*} \\
<0.001\end{array}$ \\
\hline TRACP $5 \mathrm{~b}$ (U/L) & $\begin{array}{l}\gamma \\
P\end{array}$ & $\begin{array}{c}-0.133^{*} \\
0.019\end{array}$ & $\begin{array}{c}-0.24 I^{*} \\
<0.00\end{array}$ & $\begin{array}{l}-0.228^{*} \\
<0.001\end{array}$ & $\begin{array}{c}-0.157^{*} \\
0.005\end{array}$ \\
\hline
\end{tabular}

Note: *Statistical significance at the $P<0.05$ level.

Abbreviations: BTMs, bone turnover markers; BMDs, bone mineral densities; BALP, bone-specific alkaline phosphatase; OC, osteocalcin; PINP, procollagen type I $\mathrm{N}$-terminal propeptide; $\beta$-CTX, $\beta$ cross-linked C-telopeptide of type I collagen; TRACP 5b, tartrate-resistant acid phosphatase $5 \mathrm{~b}$. 


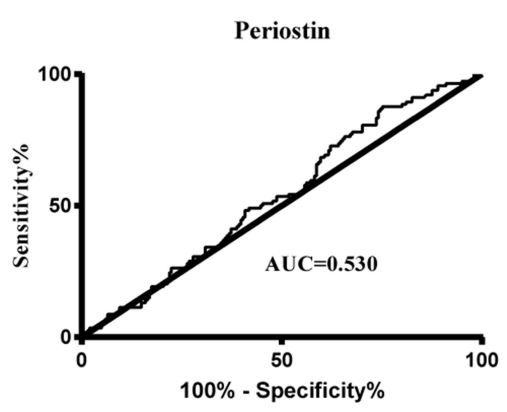

PINP

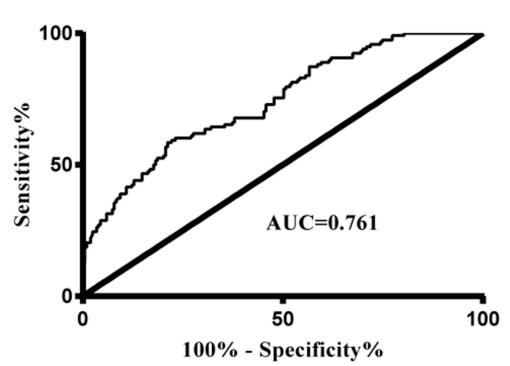

BALP

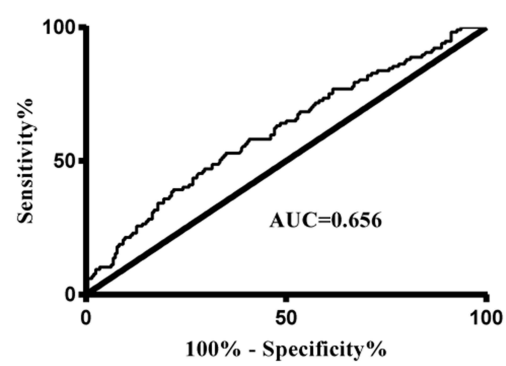

B-CTX

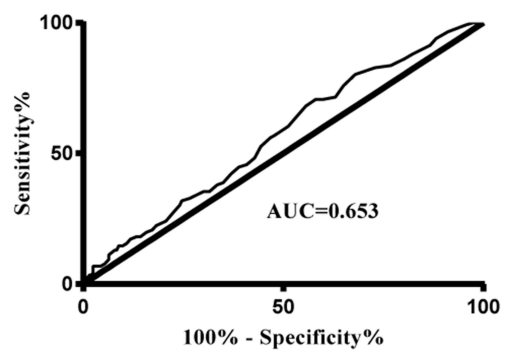

OC

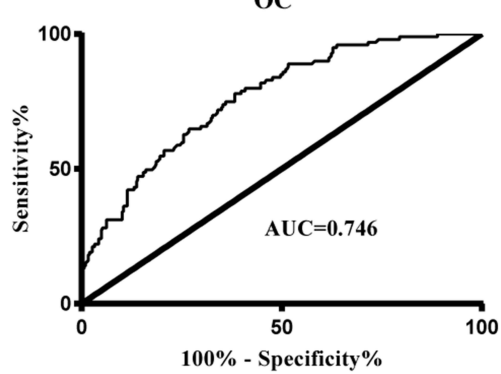

B-CTX

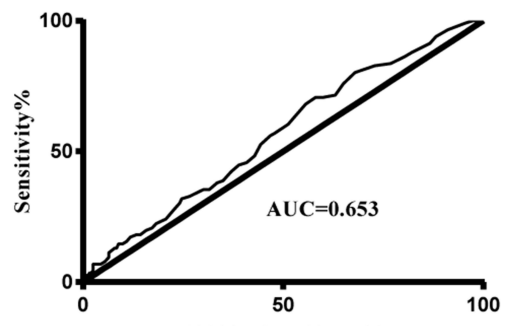

$100 \%$ - Specificity \%

Figure 2 ROC for the ability of the serum periotisin and BTMs to differentiate the postmenopausal women with osteoporosis. AUC=0.656 for BALP, $P<0.001,95 \%$ CI $0.588-0.724$; AUC $=0.746$ for OC, $P<0.001$, 95\% Cl 0.686-0.805; AUC=0.76I for PINP, $P<0.001$, 95\% Cl 0.702-0.82I; AUC=0.653 for $\beta-C T X, P<0.001$, 95\% Cl 0.587-0.7I9; AUC=0.647 for TRACP 5b, $P<0.001$, 95\% Cl 0.578-0.716; AUC=0.530 for periostin, $P=0.425,95 \% \mathrm{Cl} 0.458-0.60 \mathrm{I}$.

Abbreviations: ROC, receiver-operating characteristic curves; BTMs, bone turnover markers; AUC, area under the ROC curve; Cl, confidence interval; BALP, bonespecific alkaline phosphatase; OC, osteocalcin; PINP, procollagen type I N-terminal propeptide; $\beta$-CTX, $\beta$-cross-linked C-telopeptide of type I collagen; TRACP 5b, tartrateresistant acid phosphatase $5 \mathrm{~b}$.

bone homeostasis. ${ }^{15}$ Periostin has been reported to play a key role in bone formation and fracture healing. ${ }^{15,22,23}$ In rodent fracture models, periostin mRNA and protein expression levels are rapidly upregulated in fracture healing area. ${ }^{15}$ Therefore, periostin may be associated with bone turnover. In addition, overexpression of periostin in MC3T3-E11 osteoblastic cells increases cell adhesion and mobility. ${ }^{24}$ What's more, Li et $\mathrm{al}^{25}$ have demonstrated that $17 \beta$-estrogen promotes periostin expression at the endosteal surface and bone formation in ovariectomized rats. In view of the above basic research results, we believed that it is necessary to conduct clinical studies to observe the serum periostin levels in Chinese postmenopausal women with different bone mass.

In order to demonstrate the consistency of this studied population with the population in other studies, all traditional BTMs were also measured in this study. It was shown that all measured BTMs were negatively correlated with BMDs at different sites. These results were generally consistent with previous studies demonstrating the effectiveness of our research data. ${ }^{26,27}$

Our study found no significant difference in serum periostin levels among Chinese postmenopausal women with different bone mass, and no correlation between serum periostin levels and traditional BTMs, especially bone formation markers such as OC, BALP and P1NP. Anastasilakis et $\mathrm{al}^{18}$ have also revealed no significant increase in serum periostin level in Caucasian postmenopausal women with low bone mass $(T$-score $\leq-2.0)$ compared to the control group with normal bone mass ( $T$-score $\geq-1.0$ at the lumbar spine and the nondominant femoral neck). However, it has been observed that circulating levels of periostin were significantly increased during acute osteoporotic hip fractures in 261 Chinese older women. $^{21}$ It has been clearly known that primary bone healing after fracture is intramembrane ossification, and the bone tissue in intramembrane ossification is directly synthesized by osteoblasts, ${ }^{28}$ which may be one of the reasons for the significantly increased periostin expression in postmenopausal women after fracture. Besides this, mechanical strain may be elevated in the fracture site, which in turn stimulates the expression of periostin and promotes the bone formation. ${ }^{19,20}$ Therefore, our results that the serum periostin levels are not synchronized with traditional bone formation markers in Chinese postmenopausal women without fractures are a very interesting phenomenon. Further studies may be needed to explore the changes of periostin expression in the cortical bone 
among postmenopausal women with different bone mass and the relationship between periostin levels in the cortical bone and traditional BTMs, especially traditional bone formation markers.

The relationships between serum periostin and traditional BTMs, and BMDs at different sites in postmenopausal women remain controversial. Anastasilakis et a $1^{18}$ found that serum periostin levels were not correlated to BMD at different site and serum CTX, but positively correlated with serum BALP in postmenopausal Caucasian women. Interestingly, Kim et $\mathrm{al}^{20}$ showed that serum periostin levels in Korean postmenopausal women with non-vertebral fractures were inversely associated with all hip BMDs measured, whereas periostin levels in women with vertebral fractures were not correlated with lumbar BMD. Gossiel et $\mathrm{al}^{29}$ proved that in PMOP with teriparatide treatment, changes in periostin were unrelated to the changes in P1NP and CTX, but positively correlated with changes in the femur neck and the lumbar spine BMD.

In addition, the ROC curve analysis indicated serum PINP and OC may be the predictors of Chinese PMOP in our study. This result may provide a reference for the diagnosis of osteoporosis in countryside region of China without DEXA.

There are some limitations to the present study. Firstly, the overall number of the postmenopausal women was relatively small and unequal among the three groups. Secondly, this is a cross-sectional study, and there is no follow-up to observe the changes in serum periostin, traditional BTMs and BMD in postmenopausal women over time.

\section{Conclusions}

Although periostin is highly expressed by osteoblasts and osteocytes, there were no significant correlations between serum periostin and the classic BTMs or BMDs at different sites, suggesting that serum periostin may not reflect the overall bone metabolism in Chinese postmenopausal women without prior fracture.

\section{Abbreviations}

BTMs, bone turnover markers; BMDs, bone mineral densities; BALP, bone-specific alkaline phosphatase; OC, osteocalcin; P1NP, procollagen type $1 \mathrm{~N}$-terminal propeptide; $\beta$-CTX, $\beta$ cross-linked C-telopeptide of type I collagen; TRACP 5b, tartrate-resistant acid phosphatase 5b.DXA, dual-energy X-ray absorptiometry; ROC, the receiver-operating characteristic; WHO, World Health Organization; CV, coefficient of variation; PMOP, postmenopausal osteoporosis.

\section{Data Sharing Statement}

All data and materials have been presented in the manuscript. No outliers were omitted from the main statistical analysis. Related information is available upon request to the corresponding author.

\section{Ethics Approval and Informed Consent}

All procedures performed in studies involving human participants were in accordance with the declaration of Helsinki principles and its later amendments or comparable ethical standards. Ethical approval for the study was obtained from the Ethical Committee of Tongji Hospital of Tongji University (K-W-2021-006). Informed consent was obtained from all individual participants included in the study.

\section{Funding}

This study was sponsored by the National Natural Science Foundation of China (Grant No. 81670805) and the Science and Technology Innovation Program of Shanghai STCSM (Grant No.16411954700). All grants provided assistance for patients' follow-up and data collection.

\section{Disclosure}

The authors declare that they have no conflicts of interest for this work.

\section{References}

1. Lorentzon M, Cummings SR. Osteoporosis: the evolution of a diagnosis. J Intern Med. 2015;277(6):650-661. doi:10.1111/ joim. 12369

2. Youness ER, Mohammed NA, Morsy FA. Cadmium impact and osteoporosis: mechanism of action. Toxicol Mech Methods. 2012;22 (7):560-567. doi:10.3109/15376516.2012.702796

3. Alswat KA. Gender disparities in osteoporosis. J Clin Med Res. 2017;9(5):382-387. doi:10.14740/jocmr2970w

4. Hernlund E, Svedbom A, Ivergård $\mathrm{M}$, et al. Osteoporosis in the European Union: medical management, epidemiology and economic burden. A report prepared in collaboration with the International Osteoporosis Foundation (IOF) and the European Federation of Pharmaceutical Industry Associations (EFPIA). Arch Osteoporos. 2013;8(1-2):136. doi:10.1007/s11657-013-0136-1

5. Maggi S, Noale M, Giannini S, et al. Quantitative heel ultrasound in a population-based study in Italy and its relationship with fracture history: the ESOPO study. Osteoporos Int. 2006;17(2):237-244. doi:10.1007/s00198-005-1985-2 
6. Cui Z, Meng X, Feng H, et al. Estimation and projection about the standardized prevalence of osteoporosis in mainland China. Arch Osteoporos. 2019;15(1):2. doi:10.1007/s11657-019-0670-6

7. Kanis JA.Assessment of fracture risk and its application to screening for postmenopausal osteoporosis: synopsis of a WHO report. WHO Study Group. Osteoporos Int. 1994;4(6):368-381. doi:10.1007/ BF01622200

8. Siris ES, Chen YT, Abbott TA, et al. Bone mineral density thresholds for pharmacological intervention to prevent fractures. Arch Intern Med. 2004;164(10):1108-1112. doi:10.1001/archinte.164.10.1108

9. Schuit SC, van der Klift M, Weel AE, et al. Fracture incidence and association with bone mineral density in elderly men and women: the Rotterdam Study. Bone. 2004;34(1):195-202. doi:10.1016/j. bone.2003.10.001

10. Seibel MJ. Biochemical markers of bone turnover: part I: biochemistry and variability. Clin Biochem Rev. 2005;26(4):97-122.

11. Vasikaran S, Eastell R, Bruyère $O$, et al. Markers of bone turnover for the prediction of fracture risk and monitoring of osteoporosis treatment: a need for international reference standards. Osteoporos Int. 2011;22(2):391-420. doi:10.1007/s00198-010-1501-1

12. Chopin F, Biver E, Funck-Brentano T, et al. Prognostic interest of bone turnover markers in the management of postmenopausal osteoporosis. Joint Bone Spine. 2012;79(1):26-31. doi:10.1016/j. jbspin.2011.05.004

13. Szulc P, Garnero P, Marchand F, Duboeuf F, Delmas PD. Biochemical markers of bone formation reflect endosteal bone loss in elderly men-MINOS study. Bone. 2005;36(1):13-21. doi:10.1016/ j.bone.2004.09.004

14. Seeman E. The periosteum-a surface for all seasons. Osteoporos Int. 2007;18(2):123-128. doi:10.1007/s00198-006-0296-6

15. Bonnet N, Garnero P, Ferrari S. Periostin action in bone. Mol Cell Endocrinol. 2016;432:75-82. doi:10.1016/j.mce.2015.12.014

16. Garnero P. New developments in biological markers of bone metabolism in osteoporosis. Bone. 2014;66:46-55. doi:10.1016/j. bone.2014.05.016

17. Contié S, Voorzanger-Rousselot N, Litvin J, et al. Development of a new ELISA for serum periostin: evaluation of growth-related changes and bisphosphonate treatment in mice. Calcif Tissue Int. 2010;87(4):341-350. doi:10.1007/s00223-010-9391-y

18. Anastasilakis AD, Polyzos SA, Makras P, et al. Circulating periostin levels do not differ between postmenopausal women with normal and low bone mass and are not affected by zoledronic acid treatment. Horm Metab Res. 2014;46(2):145-149.

19. Rousseau JC, Sornay-Rendu E, Bertholon C, Chapurlat R, Garnero P. Serum periostin is associated with fracture risk in postmenopausal women: a 7-year prospective analysis of the OFELY study. $J$ Clin Endocrinol Metab. 2014;99(7):2533-2539. doi:10.1210/jc.2013-3893
20. Kim BJ, Rhee Y, Kim CH, et al. Plasma periostin associates significantly with non-vertebral but not vertebral fractures in postmenopausal women: clinical evidence for the different effects of periostin depending on the skeletal site. Bone. 2015;81:435-441. doi:10.1016/j.bone.2015.08.014

21. Farrokhi M, Arjaki D, Peykanpour F. Circulating periostin levels increase in association with bone density loss and healing progression during the early phase of hip fracture in Chinese older women. Osteoporos Int. 2020;31(10):2061. doi:10.1007/s00198-020-05606-7

22. Merle B, Garnero P. The multiple facets of periostin in bone metabolism. Osteoporos Int. 2012;23(4):1199-1212. doi:10.1007/ s00198-011-1892-7

23. Heo SC, Shin WC, Lee MJ, et al. Periostin accelerates bone healing mediated by human mesenchymal stem cell-embedded hydroxyapatite/tricalcium phosphate scaffold. PLoS One. 2015;10(3):e0116698. doi:10.1371/journal.pone.0116698

24. Horiuchi K, Amizuka N, Takeshita S, et al. Identification and characterization of a novel protein, periostin, with restricted expression to periosteum and periodontal ligament and increased expression by transforming growth factor beta. J Bone Miner Res. 1999;14 (7):1239-1249. doi:10.1359/jbmr.1999.14.7.1239

25. Li C, Li X, Wang X, et al. Periostin mediates oestrogen-induced osteogenic differentiation of bone marrow stromal cells in ovariectomised rats. Biomed Res Int. 2020;2020:9405909.

26. Chung KW, Kim MR, Yoo SW, et al. Can bone turnover markers correlate bone mass at the hip and spine according to menopausal period? Arch Gynecol Obstet. 2000;264(3):119-123. doi:10.1007/ s004040000093

27. Garnero P, Sornay-Rendu E, Chapuy MC, Delmas PD. Increased bone turnover in late postmenopausal women is a major determinant of osteoporosis. J Bone Miner Res. 1996;11(3):337-349. doi:10.1002/ jbmr.5650110307

28. Ghimire S, Miramini S, Edwards G, et al. The investigation of bone fracture healing under intramembranous and endochondral ossification. Bone Rep. 2021;14:100740. doi:10.1016/j. bonr.2020.100740

29. Gossiel F, Scott JR, Paggiosi MA, et al. Effect of teriparatide treatment on circulating periostin and its relationship to regulators of bone formation and BMD in postmenopausal women with osteoporosis. $J$ Clin Endocrinol Metab. 2018;103(4):1302-1309. doi:10.1210/jc.2017-00283
International Journal of General Medicine

\section{Publish your work in this journal}

The International Journal of General Medicine is an international, peer-reviewed open-access journal that focuses on general and internal medicine, pathogenesis, epidemiology, diagnosis, monitoring and treatment protocols. The journal is characterized by the rapid reporting of reviews, original research and clinical studies across all disease areas. The manuscript management system is completely online and includes a very quick and fair peer-review system, which is all easy to use. Visit http://www.dovepress.com/ testimonials.php to read real quotes from published authors. 